\title{
Handover parameter optimization in LTE self-organizing networks
}

\author{
Thomas Jansen*, Irina Balan ${ }^{\dagger}$, John Turk ${ }^{\ddagger}$, Ingrid Moerman ${ }^{\dagger}$, Thomas Kürner* \\ * Technische Universität Braunschweig, Braunschweig, Germany, \{jansen;kuerner $\} @$ ifn.ing.tu-bs.de \\ $\dagger$ Interdisciplinary Institute for Broadband Technology, Ghent, Belgium, \{irina.balan;ingrid.moerman\}@ intec.ugent.be \\ $\ddagger$ Vodafone Group, Newbury, England, john.turk@vodafone.com
}

\begin{abstract}
This paper presents a self-optimizing algorithm that tunes the handover (HO) parameters of a LTE (Long-Term Evolution) base station in order to improve the overall network performance and diminish negative effects (call dropping, HO failures). The proposed algorithm picks the best hysteresis and time-to-trigger combination for the current network status. We examined the effects of this self-optimizing algorithm in a realistic scenario setting and the results show an improvement from the static value settings.
\end{abstract}

\section{INTRODUCTION}

Handover is one of the key procedures for ensuring that the users move freely through the network while still being connected and being offered quality services. Since its success rate is a key indicator of user satisfaction, it is vital that this procedure happens as fast and as seamlessly as possible.

In currently deployed mobile networks, handover (HO) optimisation is done manually over a long time frame, e.g. days or weeks, on a need basis only. This approach is both time consuming and may not be carried out as often as needed. By introducing an online self-optimising algorithm that will tune the parameters of the $\mathrm{HO}$ process, we aim at overall network performance and user QoS improvement. The main targets are reducing the number of HOs that are initiated but not carried out to completion (HO failures), repeated back and forth HOs between two base stations ('ping-pong' HOs) and calls being dropped. The self-optimization of future radio access networks is one of the main topics in current research [1] [2].

Handover can be described by a very precise flow of events and some might argue that there is little if anything to be improved in it. In our approach we are looking not at modifying this flow but rather at making the parameter settings that control it flexible and modifying them accordingly. The main challenges facing such an algorithm is finding the perfect balance between the control parameters of the HO process and ensure that the network is in a stable operating point for a long time.

The rest of the paper is organized as follows. In Section II we present the assessment and simulation metrics that have been used in the simulation. The realistic SOCRATES simulation scenario is described in Section III. The optimization algorithm that tunes the handover parameters is specified in Section V. Section VI shows the simulation results and the conclusion and outlook on future work is given in Section VII.
The work has been carried out in the EU FP7 SOCRATES project [3] [4].

\section{METRICS}

The metrics that are used in the handover parameter optimisation algorithm are subdivided in system metrics, control parameters and assessment metrics. The reference signal received power (RSRP) and signal-to-interference and noise ratio (SINR) are system metrics. They are used to select the connected cell and possible handover candidates. The control parameters are tuned by the optimization algorithm to increase the handover performance of the network. The assessment metrics are used as measurements during the optimization process and as performance indicators for the optimization algorithm evaluation. The metrics are described in detail below.

\section{A. System metrics}

\section{RSRP}

The RSRP is calculated from the cell transmit power $\left(P_{c}\right)$, the pathloss values from the users to the different cells $\left(L_{u e}\right)$ and additional shadow fading with a log-normal distribution and a standard deviation of $3 \mathrm{~dB}\left(L_{f a d}\right)$. The resulting RSRP values are calculated per cell $c$ and user $u e$ by:

$$
\begin{gathered}
R S R P_{c, u e}=P_{c}-L_{u e}-L_{f a d} \\
\text { SINR }
\end{gathered}
$$

The SINR values are calculated from the RSRP of the connected cell $\left(R S R P_{\text {conn }}\right)$ and the RSRP values of the strongest interfering cells plus the thermal noise. The RSRP values of the interferers and the thermal noise are added up to ( $\left.R S R P_{\text {int,noise }}\right)$. It is assumed that all cells transmit with 46 $\mathrm{dBm}$ over the complete simulation time (transmit power taken from [8]). The resulting SINR values are calculated by:

$$
S I N R_{u e}=R S R P_{\text {conn }}-R S R P_{\text {int }, \text { noise }}
$$

\section{B. Control parameters}

A handover is initiated if two conditions are fulfilled: the RSRP of a cell is greater than the RSRP of the connected cell plus the hysteresis value and this condition holds at least for the time specified in the time-to-trigger parameter. The hysteresis and time-to-trigger will be tuned by our 
optimization algorithm.

\section{Hysteresis}

In our simulations the valid hysteresis values vary between 0 and $10 \mathrm{~dB}$ with steps of $0.5 \mathrm{~dB}$, resulting in 21 valid hysteresis values.

\section{Time-to-Trigger}

The time-to-trigger values for LTE networks are specified by 3GPP (see [5] Section 6.3.5). The values are (0 0.040 .064 $\begin{array}{llllllllllll}0.08 & 0.1 & 0.128 & 0.16 & 0.256 & 0.32 & 0.48 & 0.512 & 0.64 & 1.024 & 1.280\end{array}$ $2.5605 .120)$ in $[s]$. These 16 values are the only valid timeto-trigger values. Hence there are 336 valid control parameter combinations from the hysteresis and time-to-trigger values.

\section{Handover performance indicators (HPIs)}

\section{Handover failure ratio}

The handover failure ratio $\left(H P I_{H O F}\right)$ is the ratio of the number of failed handovers $\left(N_{\text {HOfail }}\right)$ to the number of handover attempts. The number of handover attempts is the sum of the number of successful $\left(N_{\text {HOsucc }}\right)$ and the number of failed handovers:

$$
H P I_{H O F}=\frac{N_{\text {HOfail }}}{\left(N_{\text {HOfail }}+N_{\text {HOsucc }}\right)}
$$

\section{Ping-Pong handover ratio}

If a call is handed over to a new cell and is handed back to the source cell in less than the critical time $\left(T_{\text {crit }}\right)$ this handover is considered to be a ping-pong handover. The ping-pong handover ratio $\left(H P I_{H P P}\right)$ represents the number of ping-pong handovers $\left(N_{H O p p}\right)$ divided by the total number of handovers, i.e. the number of ping-pong handovers $\left(N_{H O p p}\right)$, the number of handovers where no ping-pong occurs $\left(N_{H O n p p}\right)$ and the number of failed handovers $\left(N_{H O f a i l}\right)$ :

$$
H P I_{H P P}=\frac{N_{H O p p}}{\left(N_{H O p p}\right)+\left(N_{\text {HOnpp }}\right)+\left(N_{\text {HOfail }}\right)}
$$

\section{Call dropping ratio}

The call dropping ratio $\left(H P I_{D C}\right)$ is the probability that an existing call is dropped before it was finished, e.g. during handover, by congestion control, if the user moves out of coverage, etc. It is calculated as the ratio of the number of dropped calls ( $N_{\text {dropped }}$ ) to the number of calls that were accepted by the network $\left(N_{\text {accepted }}\right)$ :

$$
H P I_{D C}=\frac{N_{\text {dropped }}}{N_{\text {accepted }}}
$$

\section{Simulation SCEnARio}

For the handover optimization simulations we use a realistic network topology based on the network data from a network operator. The so called SOCRATES scenario also provides realistic mobility and environment data. The following information is provided by the SOCRATES scenario:

- Network configuration

- Pathloss data

- Clutter data

- Height data

- Traffic data

- Mobility data

Figure 1 shows a small section of the SOCRATES scenario. The mobiles move on the streets in the area marked with a rectangle. More details on the realistic SOCRATES reference scenario can be found in [6] and [7].

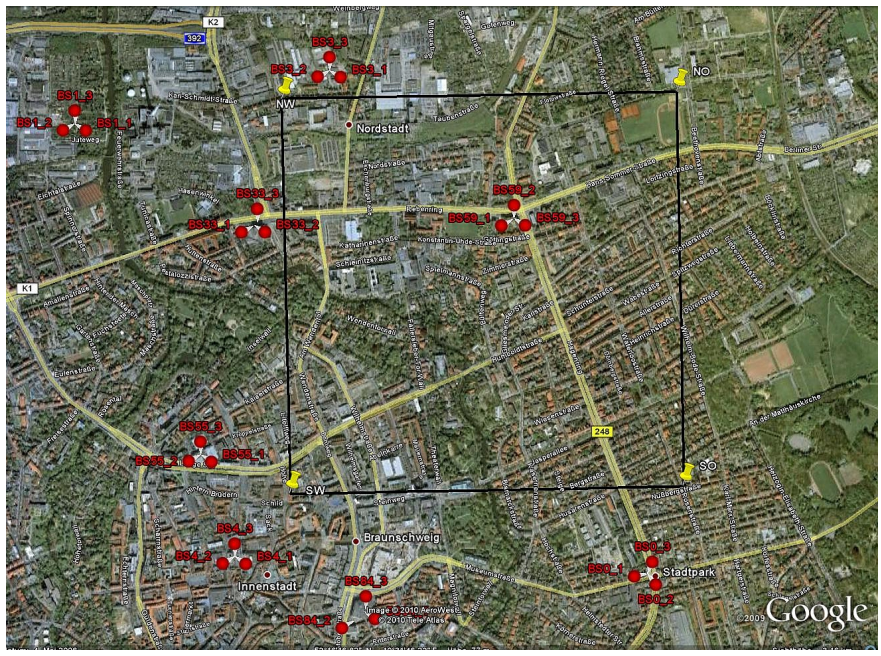

Fig. 1. The SOCRATES reference scenario

\section{SySTEM PERFORMANCE STUDIES IN THE REALISTIC NETWORK}

In the performance studies we examine the system performance of the non-optimized network for different handover parameter settings. These studies give us an insight in the effects of handover parameter changes on the system performance. From now on we will call a combination of a certain hysteresis and time-to-trigger value a handover operating point. The system performance studies are carried out for all handover operating points that are defined in Section II-B.

Figure 2 shows the simulation results for the handover performance indicator handover failures. The system simulations have been carried out for 200s simulation time for every handover operating point. The handover failure ratio is high for small hysteresis and time-to-trigger combinations. The huge amount of initiated handovers and the small hysteresis and resulting small or nonexistent SINR gain, cause the high handover failure ratio. For the HPI handover failures higher hysteresis and time-to-trigger values give a system performance without handover failures. 


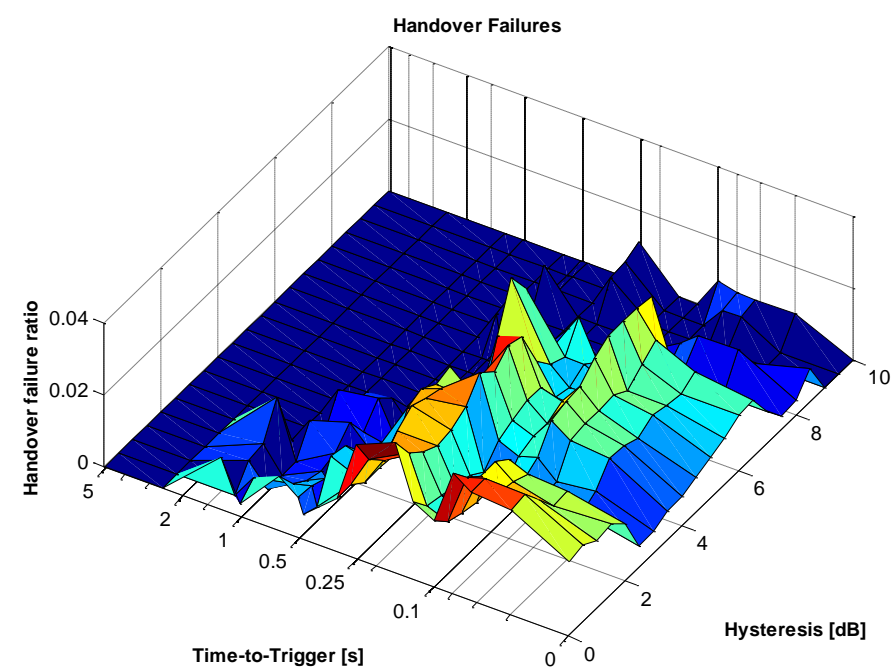

Fig. 2. Handover failure ratio in the operating points

In order to evaluate the overall system performance we have to combine the simulation results for all HPIs into one figure. Thus, the following weighting function has been defined to examine the cooperation of the HPIs:

$$
H P=\omega_{1} H P I_{H O F}+\omega_{2} H P I_{H P P}+\omega_{3} H P I_{D C}
$$

where $H P$ is the resulting handover performance, $\omega_{x}$ is the weight of the individual HPI, $H P I_{H O F}$ is the handover failure performance indicator, $H P I_{H P P}$ is the ping-pong handover performance indicator and $H P I_{D C}$ is the dropped calls performance indicator. The HPI values are normalized on the maximum value over all operating points before they are combined by the weighting function. The handover performance HP is depicted for all operating points and the weights $\left[\omega_{1}=1 \omega_{2}=0.5 \omega_{3}=2\right]$ in Figure 3.

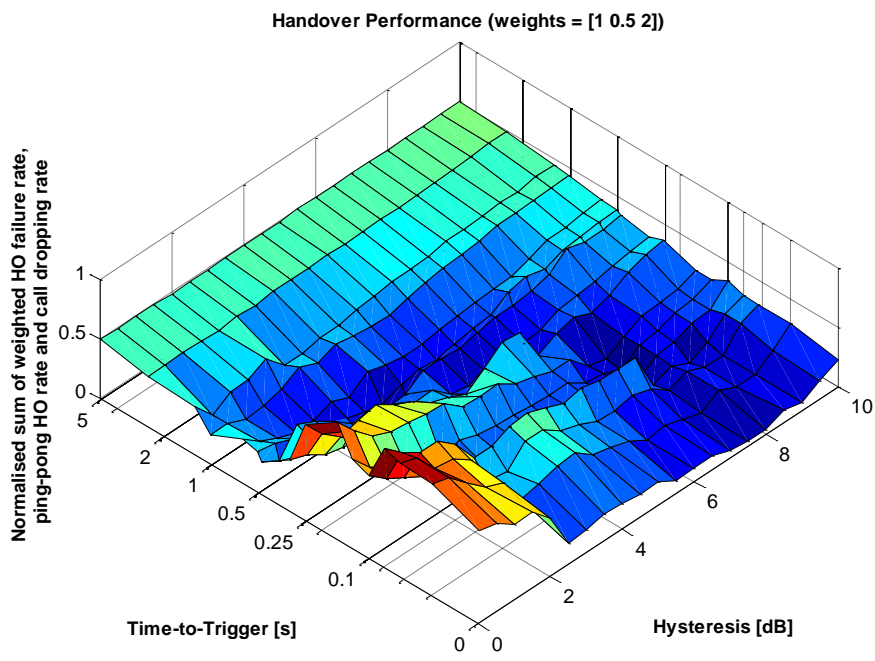

Fig. 3. Handover performance $\left(\right.$ weights $\left.=\left[\begin{array}{lll}1 & 0.5 & 2\end{array}\right]\right)$

It is normalized to the maximum handover performance value over all operating points. The different weights have been introduced to allow for operator policy inputs to the handover performance evaluation. Different operators define different sets of indicators. One operator claim could be to avoid call drops by any cost. Another operator could accept a low call dropping ratio if the signaling overhead is largely reduced. Note that for the shown system performance simulation results the handover operating points have been changed in all cells at the same time. The optimization algorithm that is explained in the next section will aim at finding the optimal handover operating points for every individual cell.

\section{HANDOVER OPTIMIZATION ALGORITHM}

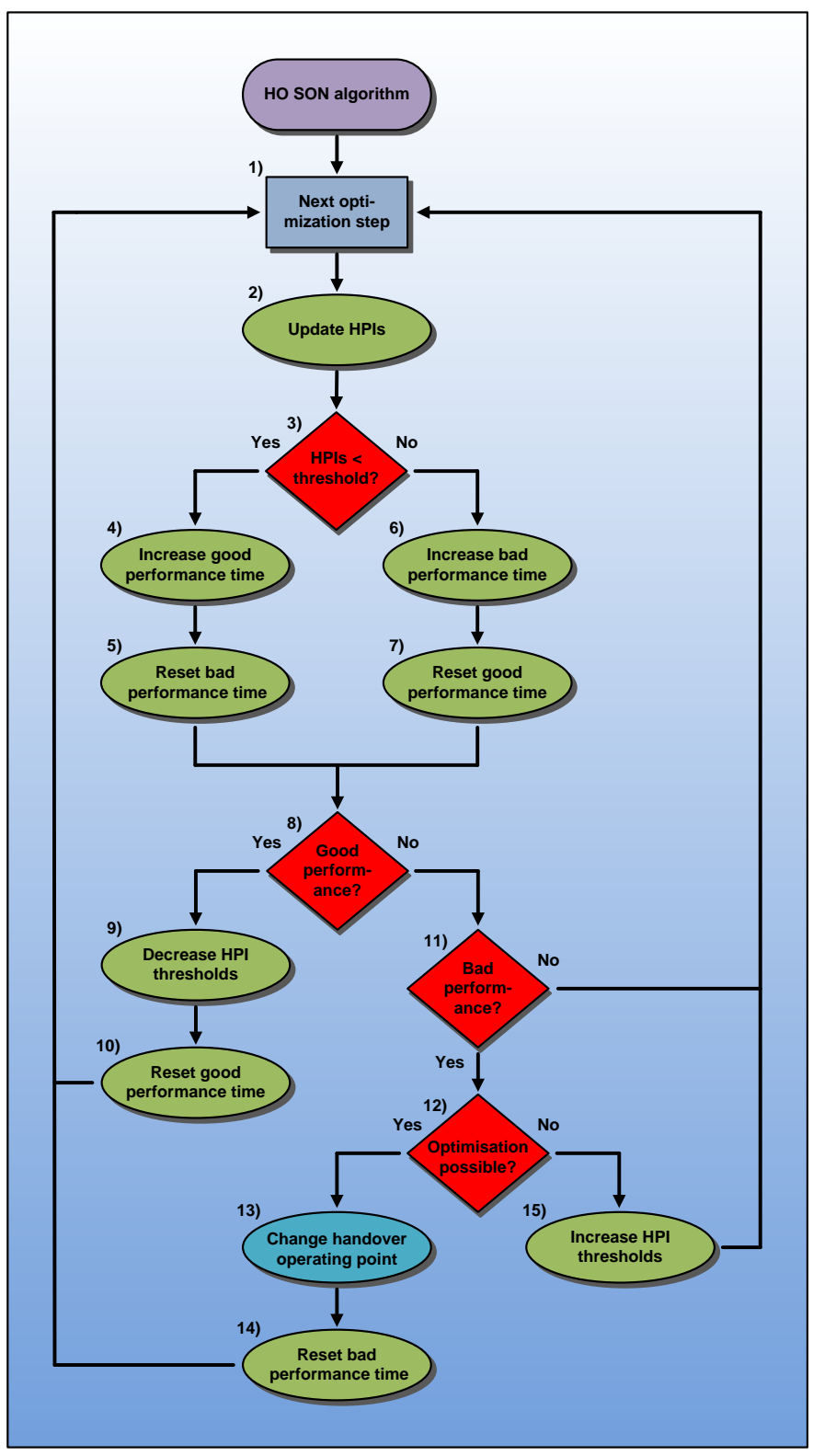

Fig. 4. The handover optimization algorithm

The handover optimization algorithm tunes the handover control parameters individually for all cells in the simulation scenario. According to the handover algorithm shown in Figure 
4 the handover operating points are changed based on the current handover performance. In order to adapt to the optimal possible handover performance we set handover performance target thresholds for all handover performance indicators, i.e. the handover failure ratio, the ping-pong handover ratio and the call dropping ratio. The target thresholds are decreased by $33 \%$ in this study if the HPIs stay below the target thresholds for a certain amount of time, called the good performance time. All simulation parameter values, like the good performance time, are given in Section VI.

The initial handover performance target thresholds are influenced by an operator policy that defines the importance of the different HPIs. If the performance of HPI call dropping ratio and one or both of the other HPIs is above the HPI target threshold, the handover performance target thresholds are increased by $33 \%$ again. The reason behind this is that the optimization criteria for these HPIs are contradictory in these cases. If the simulation parameter bad performance time exceeds a given threshold, i.e. the performance of one HPI overshoots the handover performance target for a certain amount of time, the handover operating point of the cell is changed according to the criteria given in Figure 5.

The optimization criteria are derived from system simulations for all valid operating points. The optimization direction, i.e. change of hysteresis and time-to-trigger, is based on the system performance of neighboring handover operating points. The system performance is calculated for every HPI individually. Hence the optimization direction can be lead off from these system simulations. The hysteresis value as well as the time-to-trigger values are changed by one step per handover parameter optimization only. Imagine the handover failure ratio and ping-pong handover ratio exceeded the target threshold and the optimization criteria recommend to increase the hysteresis value. The hysteresis value is only changed by one step in this case. The simulation results on the handover performance are given in the next section.

\begin{tabular}{|l|l|l|l|}
\hline $\begin{array}{l}\text { Handover } \\
\text { Performance Indicator }\end{array}$ & Hysteresis & $\begin{array}{l}\text { Time- to- } \\
\text { Trigger }\end{array}$ & Optimisation \\
\hline \multirow{2}{*}{ Handover failure ratio } & $<5 \mathrm{~dB}$ & & $\uparrow$ TTT \\
\cline { 2 - 4 } & $5 \mathrm{~dB}-7 \mathrm{~dB}$ & & $\uparrow$ TTT \& $\uparrow \mathrm{HYS}$ \\
\hline & $>7 \mathrm{~dB}$ & & $\uparrow$ HYS \\
\hline \multirow{2}{*}{$\begin{array}{l}\text { Ping-Pong handover } \\
\text { ratio }\end{array}$} & $<2.5 \mathrm{~dB}$ & & $\uparrow$ TTT \\
\hline & $2.5 \mathrm{~dB}-5.5 \mathrm{~dB}$ & & $\uparrow$ TTT \& $\uparrow \mathrm{HYS}$ \\
\hline \multirow{2}{*}{ Call dropping ratio } & $>5.5 \mathrm{~dB}$ & & $\uparrow$ HYS \\
\hline & $>6 \mathrm{~dB}$ & $>0.6 \mathrm{~s}$ & $\downarrow$ TTT \& $\downarrow$ HYS \\
\hline & $<=6 \mathrm{~dB}$ & $>0.6 \mathrm{~s}$ & $\downarrow$ TTT \\
\hline & $3.5 \mathrm{~dB}-6.5 \mathrm{~dB}$ & $<=0.6 \mathrm{~s}$ & $\uparrow$ HYS \\
\hline & $<3.5 \mathrm{~dB}$ & $<=0.6 \mathrm{~s}$ & $\uparrow$ TTT \& $\uparrow \mathrm{HYS}$ \\
\hline
\end{tabular}

Fig. 5. Optimization criteria for the handover performance indicators

\section{Simulation Results}

The system simulations are based on the simulation parameter settings shown in Figure 6. We consider 50 mobile users for these simulations that never leave the simulation area, i.e. they bounce at the scenario border. The simulation time is increased to 1000 seconds compared to the 200 seconds simulation time for the system performance simulations. The operating point with a hysteresis of $6 \mathrm{~dB}$ and a time-to-trigger of $320 \mathrm{~ms}$ is the starting operating point for all cells in the network. This handover operating point was selected since it gives a good system performance for a fixed handover operating point already.

We will later compare the handover performance of this fixed operating point to the handover performance of the optimized network. The operator policy specified in the parameter settings gives the importance of the HPIs. In this case the operator is most interested in avoiding call dropping, i.e. the operator policy value for the HPI is set to 2 .

\begin{tabular}{|c|c|}
\hline Simulation parameter & Value \\
\hline Simulation time & $1000[\mathrm{~s}]$ \\
\hline Simulation step time & $0.01[\mathrm{~s}]$ \\
\hline Simulation area (mobile users) & $1.5 \mathrm{~km} * 1.5 \mathrm{~km}$ \\
\hline Number of users & 50 \\
\hline eNodeB transmit power & $46[\mathrm{dBm}]$ \\
\hline $\begin{array}{l}\text { Operating point (Starting condition) } \\
\text { (Hysteresis, Time-to-Trigger) }\end{array}$ & $(6,0.32)$ in $[\mathrm{dB}, \mathrm{s}]$ \\
\hline $\begin{array}{l}\text { Operator policy } \\
\text { (HO failure, Ping-Pong HO, Call dropping) }\end{array}$ & $(1,0.5,2)$ \\
\hline Initial handover performance threshold & $5[\%]$ \\
\hline "good" performance threshold & $30[\mathrm{~s}]$ \\
\hline "bad" performance threshold & $10[\mathrm{~s}]$ \\
\hline Number of considered cells in the scenario & 78 \\
\hline Measured cells $(\mathrm{N})$ & 21 \\
\hline $\begin{array}{l}\text { Considered interfering cells for SINR } \\
\text { calculations }\end{array}$ & 20 \\
\hline Handover performance averaging window & $60[s]$ \\
\hline Critical ping-pong handover time (T_crit) & $5[\mathrm{~s}]$ \\
\hline Handover execution time & $0.25[\mathrm{~s}]$ \\
\hline SINR averaging window & $0.1[\mathrm{~s}]$ \\
\hline Min. SINR threshold & $-6.5[\mathrm{~dB}]$ \\
\hline
\end{tabular}

Fig. 6. Simulation parameter settings

The simulation results for the fixed operating point with a hysteresis of $6 \mathrm{~dB}$ and a time-to-trigger of $320 \mathrm{~ms}$ are depicted 
in Figure 7. The red line shows the handover failure ratio whereas the green line gives the ping-pong handover ratio. The call dropping ratio is zero for the complete simulation time. This is because the selected fixed handover operating point gives a good handover performance for the complete network already. The ping-pong handover ratio almost reaches $10 \%$ which leads to a hugh amount of signalling overhead in the network.

The optimization algorithm aims at reducing the amount of unnecessary ping-pong handovers. To examine the effect of the handover optimization algorithm the system simulations have been initiated using the same simulation parameters. The optimization algorithms starts tuning the handover parameters after 60 seconds simulation time. This is because the handover performance indicators are computed over an averaging window of 60 seconds.

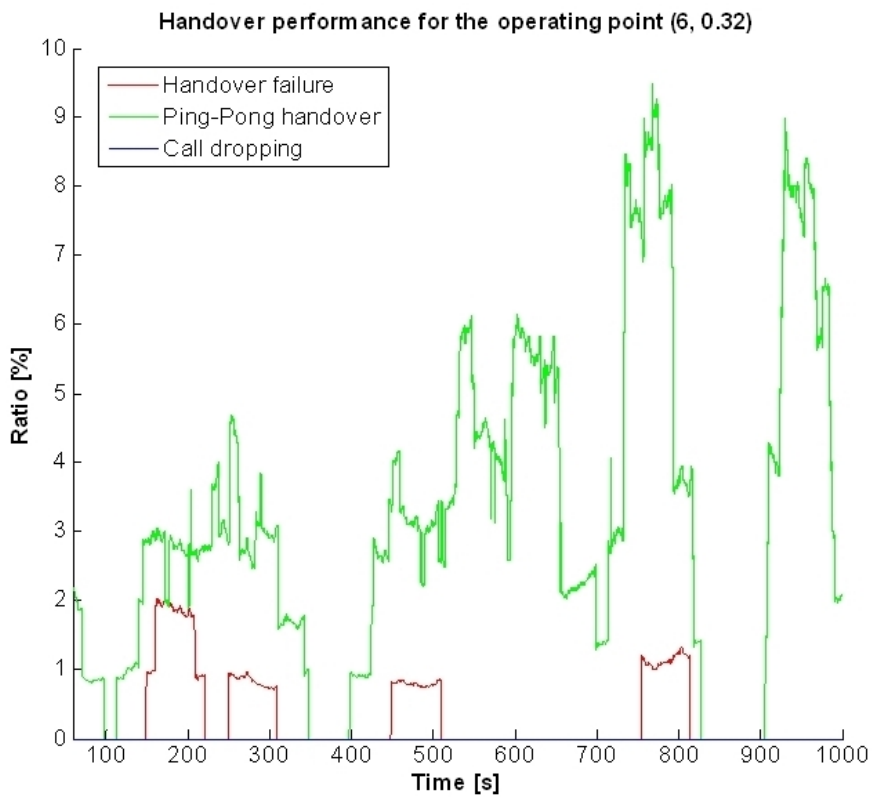

Fig. 7. Handover performance

The handover performance for the optimized network is depicted in Figure 8. The system performance is the same for the first 500 seconds simulation time. After this time the tuning of the handover parameters increases the handover performance and hence the system performance significantly. The handover failure ratio and ping-pong handover ratio are driven to zero by the optimization algorithm. The optimization activities show no negative effect on the call dropping ratio and hence satisfy the operator policy input.

\section{CONCLUSION \& OUTLOOK}

The proposed handover optimization algorithm changes the values of the hysteresis and time-to-trigger parameters in an automated manner in response to changes in the network performance. This algorithm takes into account the weighting factor given by the operator policy to different performance metrics (handover failure ratio, call dropping ratio and pingpong handover ratio).

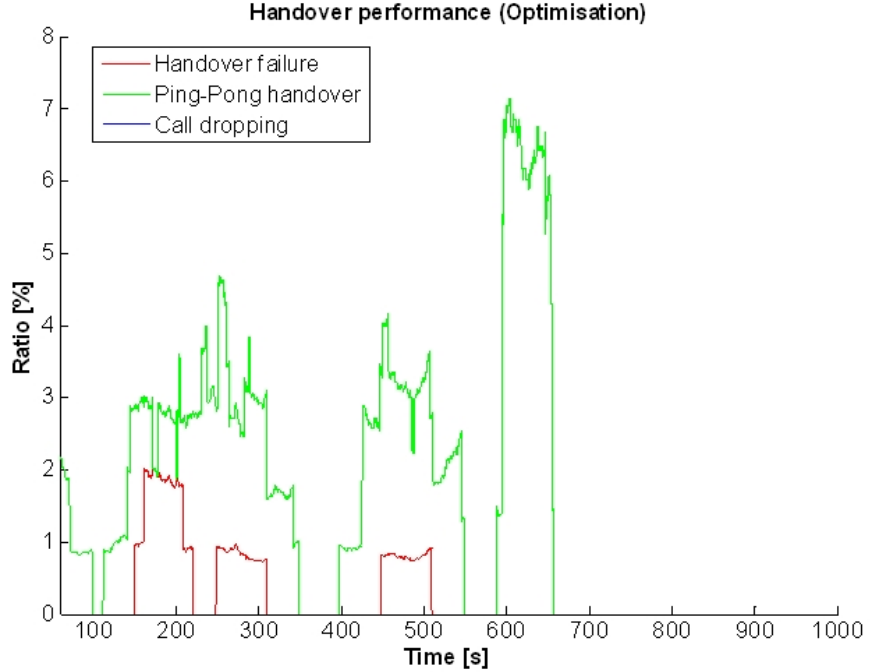

Fig. 8. Handover performance for the optimized network

This novel feature makes the SON algorithm flexible and very appealing to operators. The simulation results show that the optimization algorithm increases the system performance significantly. However the current results are limited to the used, realistic, simulation scenario. It has to be proved that the optimization algorithm works for other simulation scenarios as well.

\section{REFERENCES}

[1] 3GPP, Self-configuring and self-optimizing network use cases and solutions, Technical Report TR 36.902, available at http://www.3gpp.org.

[2] Next Generation Mobile Networks, Use Cases related to Self Organising Network, Overall Description, available at http://www.ngmn.org.

[3] SOCRATES, Self-optimisation and self-configuration in wireless networks, European Research Project, http://www.fp7-socrates.eu.

[4] M. Amirijoo, R. Litjens, K. Spaey, M. Döttling, T. Jansen, N. Scully, and U. Türke, "Use Cases, Requirements and Assessment Criteria for Future Self-Organising Radio Access Networks," Proc. 3rd Intl. Workshop on Self-Organizing Systems, IWSOS 08, Vienna, Austria, December 10-12, 2008.

[5] 3GPP, 3rd Generation Partnership Project; Technical Specification Group Radio Access Network; Evolved Universal Terrestrial Radio Access (EUTRA) Radio Resource Control (RRC); Protocol specification (Release 9), Technical Report TR 36.331 v9.1.0, available at http://www.3gpp.org.

[6] N. Scully, J. Turk, R. Litjens, U. Türke, M. Amirijoo, T. Jansen and L. C. Schmelz Review of use cases and framework II, Deliverable 2.6 EU-Project SOCRATES, December 2009.

[7] H. Schumacher, M. Schack and T. Kürner, "Coupling of Simulators for the Investigation of Car-to-X Communication Aspects" 7th COST2100 Management Committee Meeting, TD(09)773, Braunschweig, Germany, February 2009

[8] 3GPP, 3rd Generation Partnership Project;Technical Specification Group Radio Access Network;Evolved Universal Terrestrial Radio Access (EUTRA);Radio Frequency (RF) system scenarios (Release 8), Technical Report TR 36.942, available at http://www.3gpp.org. 